\title{
Preparation, Linear Spectrum and Nonlinear Two-photon Absorption Properties of Fluorenone Derivative Containing 4-Methoxystyrene
}

\author{
Shu-Heng $\mathrm{CHI}^{1, \mathrm{a}}$, Liang $\mathrm{LI}^{1, \mathrm{~b}}$, Yi-Qun $\mathrm{WU}^{1,2, \mathrm{c}^{*}}$ \\ ${ }^{1}$ Key Laboratory of Material Science and Technology for High Power Lasers, Shanghai Institute of \\ Optics and Fine Mechanics, Chinese Academy of Sciences, Shanghai 201800, PR China \\ ${ }^{2}$ Key Lab of Functional Inorganic Material Chemistry (Heilongjiang University), Ministry of Education, \\ Harbin 150080, PR China \\ achshhen@163.com, bbrightlee@siom.ac.cn, 'yqwu@siom.ac.cn \\ ${ }^{*}$ Corresponding author
}

Keywords: Preparation, Linear Spectrum, Nonlinear Two-photon Absorption, Fluorenone Derivative.

\begin{abstract}
The nonlinear optics is a new and important branch in the field of optical physics. Compared with the traditional optics, the nonlinear optics can produce many special phenomena such as frequency doubling, optical limiting, two-photon absorption and so on. Therefore, it has great potential for application in high technology. In this research, A novel fluorenone derivative containing 4-methoxystyrene with symmetric structure $\mathrm{D}-\pi-\mathrm{A}-\pi-\mathrm{D}$ is prepared using the heck reaction, namely, 2,7-bis((E)-4-methoxystyryl)-9H-fluoren-9-one(W-J-B). The linear spectrum including ultraviolet absorption spectra and one-photon fluorescence emission spectra are measured, the nonlinear two-photon fluorescence emission spectra is researched in different excitation wavelength and powers. The results exhibit that this novel organic material has good two-photon absorption performance and nonlinear optical effect, which has potential application prospect in the field of new and high technology such as frequency up-conversion, 3D micro-fabrication, photodynamic therapy, 3D optical data storage and so on.
\end{abstract}

\section{Introduction}

Two-photon absorption (2PA) is a kind of three order nonlinear optical process. Two-photon absorption has good three-dimensional spatial selectivity, high penetration depth, long-wave excitation, shortwave emission and other advantages[1-3]. The two-photon absorption cross-section is a important physical parameter, which reflects the strength of abilities for molecular to absorb two photons. Therefore, research and development of organic two-photon absorption materials with large two-photon absorption cross sections, high fluorescence quantum yield, obvious nonlinear optical effect, good photo-physical and photochemical stability in recent years became a hot topic in the field[4-6]. The organic materials studied for 2PA properties in previous research involved lots of types of conjugated structures with acceptor and donor groups such as benzene, pyridine, diphenylamine, triphenylamine and so on. In order to increase the 2PA cross section, most of previous researchers focused on increasing the molecular chain length, enlarging conjugated delocalized plane and improving the efficiency of charge transfer[7-9]. However, the majority of 2PA materials existed some defects in the performance such as poor chemical stabilities, smaller 2PA cross section and lower fluorescence quantum yield, which limited the application prospect of two-photon absorption materials in the field of high technology[10-12]. Therefore, a novel symmetric D- $\pi-A-\pi-D$ organic material 2,7-bis((E)-4-methoxystyryl)-9H-fluoren-9-one(W-J-B) was designed and prepared based on the relationship between molecular structure and properties in this research. The 2,7-dibromo-9H-fluoren-9-one was selected as the electron acceptor group due to its biphenyl structure with rigid planarity, ethylene units acted as $\pi$-bridge, and 4-methoxystyrenes were connected symmetrically on both sides of the $\pi$-conjugated bridge, which could effectively improve the conjugation length and intra-molecular charge transfer (ICT) as the electron donor groups. The 
linear absorption and fluorescence emission spectra were studied, the two-photon excitation fluorescence (TPEF) spectra was investigated in different excitation wavelength and power.

\section{Experimental Details}

2,7-dibromo-9H-fluoren-9-one, 4-methoxystyrene, tri(o-tolyl)phosphine, anhydrous potassium carbonate and palladium acetate were purchased and used as received. All the reagents and solvents were analytical grade. The target compound W-J-B was synthesized using heck reaction. The synthetic reaction route and molecular structure of the target compound W-J-B were shown in Fig.1.

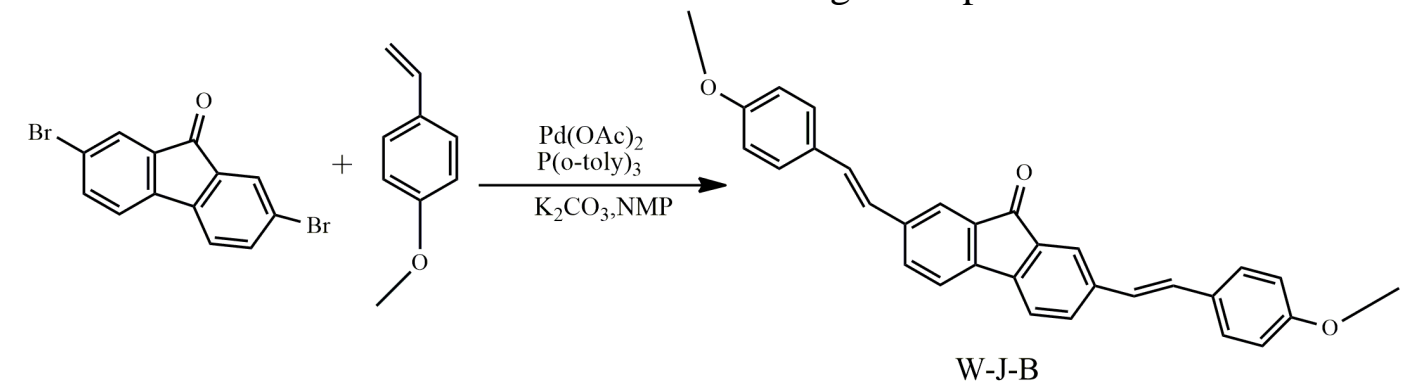

Fig.1 The synthetic reaction route of the target compound W-J-B

Synthesis of 2,7-bis((E)-4-methoxystyryl)-9H-fluoren-9-one: The 2,7-dibromo-9H-fluoren-9-one was added to a three-neck-flask with a mixture of 4-methoxystyrene, tri(o-tolyl)phosphine, anhydrous potassium carbonate, palladium acetate, and then amount of N-methyl-2- pyrrolidone(NMP) was added to this flask. Then the container was heated to $130{ }^{\circ} \mathrm{C}$ under the protection of nitrogen. After keeping this system for $8 \mathrm{~h}$, the turbid liquid was extracted and separated by organic solvent, then the product W-J-B was collected as a red powder by vacuum distillation and column chromatography on silica gel.

Yield 46.5\%. ${ }^{1} \mathrm{HNMR}(\mathrm{DMSO}): \delta(\mathrm{ppm}) 3.79\left(3 \mathrm{H}, \mathrm{CH}_{3} \mathrm{O}\right) ; 7.0(2 \mathrm{H}, \mathrm{CH}=\mathrm{CH}) ; 7.2-7.4(4 \mathrm{H}$,phenyl ring); 7.6-7.86(3H,fluorenone). IR(KBr): 2959, 2836, 1717, 1512, 1250, 1030, 978, 952, 835, 830; MALDI-TOF-MS: m/z, 444.17(444.23)[M].

\section{Linear Spectrum Measurements}

The linear absorption spectrum was measured with a Perkin-Elmer Lambda 9 UV/VIS/NIR spectrometer. The influence of quartz cuvette and solvent had been deducted. The fluorescence spectrum was measured using FP-6500 fluorescence spectrometer at room temperature. While the fluorescence quantum yield for the compound W-J-B in DMF was calculated using the same concentration of Rhodamine B in ethanol at $25^{\circ} \mathrm{C}(\Phi=0.9)$ as a standard. The calculation formula of the fluorescence quantum yield as follows (Eq. 1):

$$
\Phi_{s}=\Phi_{r} \times \frac{\int F_{s}}{\int F_{r}} \times \frac{A_{r}}{A_{s}} \times \frac{n_{s}^{2}}{n_{r}^{2}}
$$

The measurement concentration of linear and fluorescence spectra was at a concentration of $10^{-5} \mathrm{M}$.

\section{Nonlinear Two-photon Optical Properties Measurements}

The two-photon optical properties are measured using Ti: sapphire laser as the pump source. For the measurement of two-photon excited fluorescence (TPEF) spectra under different pumped power at a concentration of $10^{-4} \mathrm{M}$, The working parameters are as follows: the pulse width of $120 \mathrm{fs}$, the repetition rate of $1 \mathrm{KHz}$ and the center wavelength of $800 \mathrm{~nm}$. For the measurement of two-photon excited fluorescence spectra under different excitation wavelength, the Ti: sapphire laser $(750-850 \mathrm{~nm}$, $80 \mathrm{MHz}, 80 \mathrm{fs}$ pulse width) is used. 
The two-photon absorption cross-sections are measured by the two-photon excited fluorescence method. The Rhodamine B in ethanol is selected as the reference at a concentration of $10^{-4} \mathrm{M}$, the $2 \mathrm{PA}$ cross-section of the reference comes from the literature[13,14]. The 2PA cross-section of the target compound is determined by calculation formula (Eq. 2):

$$
\sigma_{s}=\sigma_{r} \times \frac{F_{s}}{F_{r}} \times \frac{\Phi_{r}}{\Phi_{s}} \times \frac{C_{r}}{C_{s}} \times \frac{n_{r}}{n_{s}}
$$

Where the " $r$ " stand for the reference sample, " $s "$ stand for the sample. $\delta$ is the 2PA cross-section, $\Phi$ is the fluorescence quantum yield, $F$ is the two-photon fluorescence integral intensity, $c$ is the concentration of solutions, $n$ is the refractive index of solutions.

\section{Results and Discussion}

The UV absorption spectra and fluorescence emission spectra of the fluorenone derivative W-J-B were shown in Fig.2.The One-photon fluorescence properties of W-J-B were shown in Tab.1.It can be seen that the maximum absorption wavelength and the maximum excitation wavelength are at $374 \mathrm{~nm}$ and $376 \mathrm{~nm}$ respectively. While the fluorescence maxima is at $521 \mathrm{~nm}$, locating the green spectral range. The stokes shift was $147 \mathrm{~nm}$, which may be due to intra-molecular resonance effect $[15,16]$. The fluorescence quantum yield is 0.386 , which indicated in a certain extent that the introduction of symmetric D- $\pi$-A- $\pi$-D structure lead to more homogeneous distribution density of electron cloud in the molecular conjugated system [17].

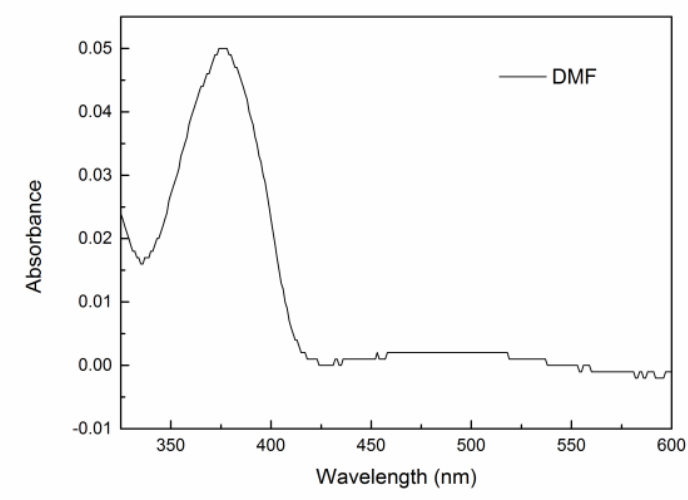

(a)

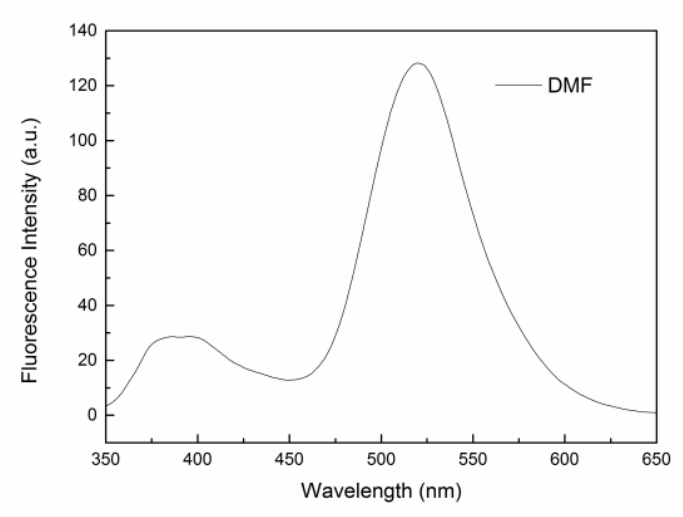

(b)

Fig.2 The UV absorption (a) and fluorescence emission spectra (b) of the sample in DMF solution

Tab.1 One-photon fluorescence properties for the material W-J-B in DMF solution

\begin{tabular}{cccccc}
\hline Compound & $\lambda_{\text {abs }}(\mathrm{nm})$ & $\lambda_{\text {ex }}(\mathrm{nm})$ & $\lambda_{\text {em }}(\mathrm{nm})$ & $\Delta \lambda_{\text {Stokes }}(\mathrm{nm})$ & $\Phi$ \\
\hline W-J-B & 374 & 376 & 521 & 147 & 0.386 \\
\hline
\end{tabular}

The two photon fluorescence spectra of the fluorenone derivative $\mathrm{W}-\mathrm{J}-\mathrm{B}$ in DMF solution at different pumped power (excited by $750 \mathrm{~nm}$ wavelength) were researched. The logarithmic relationship between incident intensity $\left(\mathrm{I}_{\mathrm{O}}\right)$ and fluorescence intensity $\left(\mathrm{I}_{\mathrm{F}}\right)$ is shown in Fig. 3. The slope of the line was 1.98 , which was closed to 2 . This confirmed that the up conversion luminescence of the DMF solution of the material W-J-B is caused by two-photon absorption process. Furthermore, the wavelength positions of two-photon fluorescence and single-photon fluorescence were both 
located in the range of 515-525 nm, which further proved that the fluorescence that the fluorenone derivative $\mathrm{W}-\mathrm{J}-\mathrm{B}$ produced in the relaxation process was the same.

The obtained 2PA cross-sections of the fluorenone derivative W-J-B from $730 \mathrm{~nm}$ to $790 \mathrm{~nm}$ at intervals of $10 \mathrm{~nm}$ are shown in Fig. 4 and Tab.2. It can be seen that, when the excitation wavelength is $750 \mathrm{~nm}$, the maximum 2PA cross-section is $727.43 \mathrm{GM}$. This demonstrated that the optimal two-photon excitation wavelength of W-J-B is $750 \mathrm{~nm}$ at the same pumped power. Obviously, the rigid biphenyl structure of the fluorenone and the introduction of symmetric donor groups lead to the large TPA cross-sections at different excitation wavelength. Therefore, the fluorenone derivative produced relatively strong and obvious up-conversion fluorescence and nonlinear optical effect in near infrared femto-second laser excitation, which implied that this material could have good application prospect in the field of optical power limiting, 3D micro-fabrication and 3D optical storage, etc.
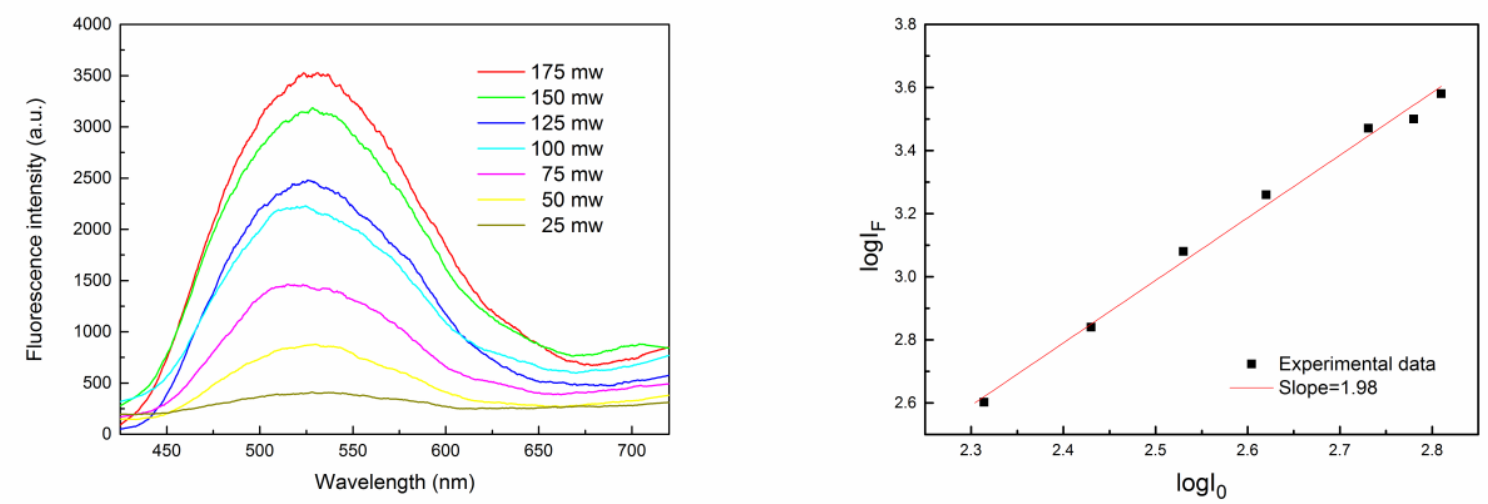

Fig.3 TPEF properties of W-J-B in DMF solution at different pumped power

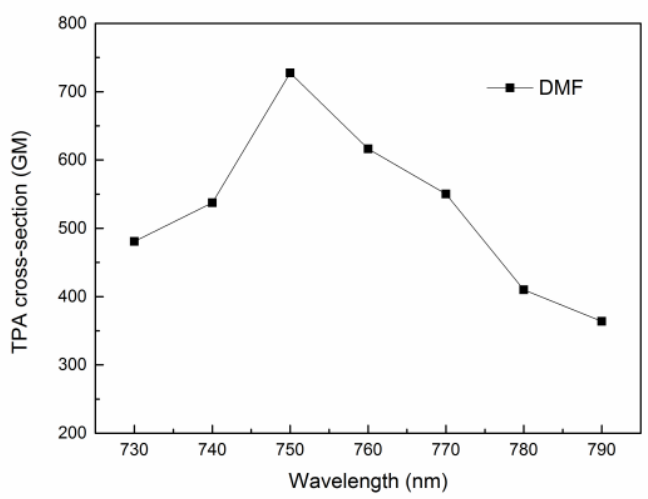

Fig.4 TPA cross-sections of W-J-B in DMF solution from $730 \mathrm{~nm}$ to $790 \mathrm{~nm}$

Tab.2 The data of TPA cross-sections for W-J-B in DMF solution from $730 \mathrm{~nm}$ to $790 \mathrm{~nm}$

\begin{tabular}{llllllll}
\hline & $730 \mathrm{~nm}$ & $740 \mathrm{~nm}$ & $750 \mathrm{~nm}$ & $760 \mathrm{~nm}$ & $770 \mathrm{~nm}$ & $780 \mathrm{~nm}$ & $790 \mathrm{~nm}$ \\
\hline$\delta_{T P A}(G M)$ & 480.77 & 537.42 & 727.43 & 616.15 & 550.1 & 410.04 & 363.84 \\
\hline
\end{tabular}

\section{Conclusion}

In summary, A novel fluorenone derivative with symmetric $D-\pi$-A- $\pi$-D type structure, 2,7-bis((E)-4-methoxystyryl)-9H-fluoren-9-one, is prepared using heck reaction. The maximum 
absorption is at $374 \mathrm{~nm}$. The maximum fluorescence emission wavelength is $521 \mathrm{~nm}$. The Stokes shift reaches $147 \mathrm{~nm}$ due to intra-molecular resonance effect. The fluorescence quantum yield reached 0.386 as a result of the homogeneous distribution density of electron cloud in the molecular structure. The maximum 2PA cross-section reaches $727.43 \mathrm{GM}$ at the excitation wavelength of $750 \mathrm{~nm}$. All the above results show that this material with good linear and nonlinear photo-physical properties may have potential application value in optical power limiting, 3D micro-fabrication and 3D optical storage, etc.

\section{Acknowledgement}

This work was partially supported by the National Natural Science Foundation of China (Grant Nos. 61137002, 61178059 and 51172253).

\section{References}

[1]W. Zhou, S.M. Kuebler, K.L. Braum, T. Yu, J.K. Cammack, C.K.Ober, J.W. Perry, S.R. Marder, Science 296 (2002) 1106.

[2]A. Abbotto, L. Beverina, R. Bozio, A. Facchetti, C. Ferrante, G.A.Pagani, D. Pedron, R. Signorini, Org. Lett. 4 (2002) 1495.

[3]D.J. Pikas, S.M. Kirkpatrick, D.W. Tomlin, L. Natarajam, V.Tondrglia, T.J. Bumming, Appl. Phys. A 74 (2002) 767.

[4]Hye Jeong Lee, Jiwon Sohn, Jaehoon Hwang, Soo Young Park, Haeyoung Choi, Myoungsik Cha, Chem. Mater. 16 (2004) 456.

[5]G.S. He, L.-S. Tan, Q.D. Zheng, P.N. Prasad, Chemical Reviews 108 (2008) 1245.

[6]J.-Z. Cheng, C.-C. Lin, P.-T. Chou, A. Chaskar, K.-T. Wong, Tetrahedron 67 (2011)734.

[7]Pond SJK, Rumi M, Levin MD, Parker TC, Beljonne D, Day MW, et al. J Phys Chem A 2002;106: 11470-80.

[8]Woo HY, Liu B, Kohler B, Korystov D, Mikhailovsky A, Bazan GC. J Am Chem Soc 2005;127: 14721-9.

[9]Fitilis I, Fakis M, Polyzos I, Giannetas V, Persephonis P, Vellis P, et al. Chem Phys Lett 2007;447: 300-4.

[10]Cao DX, Liu ZQ, Zhang GH, Li GZ. Dyes and Pigments 2009;81:193-6.

[11]Lartia R, Allain C, Bordeau G, Schmidt F, Fiorini-Debuisschert C, Charra F, et al. The Journal of Organic Chemistry 2008;73:1732-44.

[12]Xiao HB, Leng B, Tian H. Polymer 2005;46:5707-13.

[13]Xu C, Webb W.W. J. Opt. Soc. Am. B, 13, 481 (1996).

[14]Wolleschensky R., Feurer T., Sauerbrey R., Simon U. Appl. Phys.B., 67, 87 (1998).

[15]A.K.-Y. Jen, V.P. Rao, K.Y. Wong, K.J. Drost, J. Chem. Soc. Chem. Commun (1993) 90.

[16]G. Hughes, D. Kreher, C.S. Wang, A.S. Batsanov, et al., Org. Biomol. Chem. 2(2004) 3363-3367.

[17]Diaspro, A.; Federici, F.; Viappiani, C.; Krol, S.; Pisciotta, M.;Chirico, G.; Cannone, F.; Gliozzi, A. J. Phys. Chem. B 2003, 107, 11008. 